\title{
Novel Mechanism of Classifying the Brain Tumor for Identifying its Critical State
}

\author{
Deepthi Murthy T S \\ Assistant Professor \\ School of ECE, REVA University, \\ Bangalore, India
}

\author{
Sadashivappa G \\ Professor \\ Department of Telecommunication \\ Engineering, RV College of \\ Engineering, Bangalore, India
}

\author{
Ravi Shankar D \\ Assistant Professor \\ School of ECE, REVA University, \\ Bangalore, India
}

\begin{abstract}
Classification of brain tumor is one of the most challenging tasks in the clinical and radiological research. Upon investigating the existing research contribution, we find that still there is wide open scope of addressing classification problem pertaining to brain tumor. Therefore, this manuscript presents a simple mechanism of classifying the brain tumor in order to categorize its state of criticality. The proposed system applies a multi-level preprocessing to enhance the input image followed by image thresholding for feature extraction and decomposition using wavelet transform. The extracted features are further subjected to process of dimensional reduction that maintains a balance between good number of enriched feature and less size of redundant feature using statistical approach. Further, a supervised learning approach is implemented that further optimizes the classification process. The study outcome is further benchmarked with different process of classification to show the efficient computational environment of proposed system.
\end{abstract}

Keywords-Brain tumor; classification; categorization; clustering; identification; segmentation; MRI; Brain X-Ray

\section{INTRODUCTION}

Brain tumor is considered to be a dreadful clinical condition affecting both patient and doctor to deal with. It is basically an abnormal growth of tissues which could be either lethal (malignant) or non-lethal (benign). However, there are also fair chances of transformation of non-lethal to lethal tumors in many cases. At present, with the advancement of medical imaging, it is now not a big deal to obtain the imaging of the brain tumor [1]. Magnetic Resonance Imaging or popularly known as MRI is one of the best mechanism to obtain multi-dimensional as well as discrete visualization of brain tumor [2]. However still with such advance imaging, there are significant problems associated with both identification of tumors as well as classifying it. The first problem associated with tumor detection is illumination factor [3]. Variable illumination factors e.g. abnormal brightness, contrast, saturation, etc may significant affect the detection rate. Similarly, there are other set of problems associated with classifying what sort of tumor it is. The prime reason behind such classification problem is many. Normally, there are neoplastic tissues in brain that are considered to be nonhomogeneous in its natural state when the imaging is taken [4]. Apart from this, such tissues are also found to be overlapped with each other as found in the imaging that poses a significant level of challenge for the physician to actually even understand the type of tumor. The normal tissues are also found to be overlapped with such neoplastic tissues making it further impossible even to identify that is it a tumor or normal tissue. On the other hand, there is another type of tumor called as Glioma that associated with various types of brain cells. It will mean that Gliomas exhibit multiple characteristics in imaging making it more difficult to classify. In order to solve such problem, the medical community has evolved up with a reference model of brain neoplasm that considered analysis based on histopathological images [5]. This process is further confirmed by surgical biopsy. In simpler way, there is no straight forward imaging technique of classification of brain tumor although identification of tumor is somewhat feasible based on skills of radiologist.

In the last few decades, there have been certain serious research attempts toward exploring classification problems [6][10]. Majority of these existing research contributions has been focusing more on detection and segmentation rather than on classification problems. A closer look into the problems associated with the existing research work are i) more work towards detection and less towards classification, ii) few studies with benchmarked outcomes, iii) usage of region of interest, iv) consistent usage of machine learning approach with computational complexity. Hence, all these above problems are essential to be mitigated as the present demand of the application is not only accuracy but also computational friendly application. At present, software used for diagnosis are modeled to run on low-power and low-resourceful computing mobile device. Hence, there is a need to evolve up with computational model that can balance both the classification performance as well as computational efficiency. Therefore, the proposed manuscript introduces a simple and yet novel classification process to determine the malignant and benign case of brain tumor. The paper also outlines the simple usage of feature detection, detection, dimensional reduction, and learning involved. Section II highlights existing research works in tumor classification followed by problems associated with existing system are discussed in Section III. Section IV briefs the proposed research methodology and Section $\mathrm{V}$ gives the algorithm design while the results analysis described in Section VI. Finally, Section VII summarizes the proposed system.

\section{RELATED WORKS}

This section discusses about research contribution of existing authors towards addressing the problems associated with the classification of the tumor in medical images. 
Bahadure et al. [11] have emphasized on performing segmentation technique to ensure better detection performance of brain tumors using wavelet transform and supervised learning approach. Most recently a classification approach using ensemble classifier is introduced by Cong et al. [12] where multiple clustering techniques has been used for diagnosis cancerous section. Usage of ensemble classifier was also reported in the work carried by Esener et al. [13]. The author have used region-of-interest followed by preprocessing and feature extraction for introducing classification of multistage cancer. Jadoon et al.[14] have adopted deep learning method in in order to perform classification. The author have also used curvelet-based transform as well as adaptive histogram and wavelet transform in the preliminary stages followed by training using convolution neural network and support vector machine. Ravi et al. [15] have emphasized on the segmentation scheme for enhanced detection of abnormal region in brain imaging. The authors have used stochastic approach as well as semantic approach for performing classification. Adoption of deep learning approach was also seen in the work of Song et al. [16] towards solving classification problems associated with radiological images. Vu et al. [17] have presented a dictionary learning technique for effective classification of histopathological images. The scheme assists in generation of rich number of features from brain tumor images. Xi et al. [18] have implemented swarm intelligence and supervised learning technique for enhancing the selection process of feature that directly assisted in solving classification problems. Zhao and Jia [19] have advocated the use of convolution neural network that is claimed to have its superior effect in segmentation performance. The scheme is also said to maintain equal emphasis on both local and global region.

Usage of neural network was also reported in the work carried out by Marcomini et al. [20]. The authors have used self-organizing map along with multiple segmentation process assisting in better classification process. Barbalata and Mattos [21] have used anisotropic filtering mechanism in order to perform detection and classification of various critical lesions on the radiological image. Anitha and Murugavalli [22] have enhanced k-means algorithm to incorporate adaptiveness in order to assist in solving segmentation and classification problems. The authors have also used neural network and wavelet transform. Kaur et al. [23] have introduced the usage of ensemble classifier as well as Hilbert transformation for enhanced representation of radiological image. The implemented algorithm was proven to have maximum accuracy level on brain tumor images. Jui et al. [24] have emphasized on the feature extraction scheme for enhancing the segmentation process as well as classification process all together. Emphasis on feature selection process is also carried out by Huda et al. [25], where the authors have morphometric analysis of radiological images followed by applying ensemble based classifier. Svensson et al. [26] have investigated on supervised classification technique as well as random forest in order to evolve up with a better classification scheme. Work carried out by Sharma and Marikkannu [27] have introduced as unique classification technique. The authors have used Gaussian filter in preliminary state followed by a nonconventional mechanism of extracting features based on distribution of gray level. Further optimization of features were carried out by search algorithm followed by series of learning algorithms e.g. support vector machine, back propagation, knearest algorithm etc to obtain better classification performance. Automation process in extraction of significant feature assists in precise identification of tumors. This fact has been proven by Chadded [28] by using Gaussian mixture model. Bukovsky et al. [29] have used neural network in order to perform identification followed by prediction of tumor. This process is a way advanced to classification process using feedforward neural network. Acevedo et al. [30] have implemented memory-based scheme of bidirectional nature in order to increase the accuracy of classification process. Kothavari et al. [31] have used machine learning approach along with search optimization in order to emphasize on the feature extraction process. Chen and Jian [32] have used regression approach for carrying out classification of tumor. Huang et al. [33] have introduced a projection-based mechanism as well as regression for assisting in solving classification problems. The technique assists in categorizing voxels of clinical importance and thereby emphasizing on the locality factor of the given radiological image. Huml et al. [34] have used genetic programming along with the usage of morphological marker in order to assists in classification of brain tumor. The technique further introduces usage of mining approaches in order to extract more precise clinical information directly assisting in tumor classification. Corso et al. [35] have used Bayesian classification approach for localizing the brain tumor. Saddique et al. [36] have used a contour-based technique in order to identify the symmetry factor followed by segmentation process. Nanthagopal and Sukanesh [37] have used waveletbased technique for feature extraction and used both support vector machine and neural network for classification of brain tumor. Sun et al. [38] have implemented a learning approach using support vector machine and Eigen value for classification of tumor. The technique also uses independent component analysis. Usage of neural network was reported in the work of Brougham et al. [39] on spectroscopic data for solving its associated classification problems. Usage of matrix factorization is adopted in the work of Zheng et al. [40] for solving similar problems associated with tumor classification. The behavior of tumor is found in Astana and Vashith [41] in which brain MRI is considered for defining the criticality of tumor. In [41], time taken by manual segmentation is minimized by using appropriate quantitative technique which can be useful for futuristic clinical research. The performance analysis of [41] states significant outcomes. The next section briefs up the research problem associated with existing research work.

\section{PROBLEM IDENTIFICATION}

From the prior section, it can be seen that there has been various research techniques work on solving classification problems. All the problems offers advantages as well as is also associated with certain unsolved problems e.g. i) existing techniques are more inclined towards machine learning algorithms where the proportion of learning operation is too much complex and more as compared to other lightweight operations. This process although shows good increase in accuracy but they are highly subjective in nature. It will mean 
that such algorithms don't offer similar performance when the environmental variables are changed, ii) existing techniques also doesn't emphasizes on pre-processing steps. All the existing techniques apply any one preprocessing without knowing if they are really suitable or not, iii) existing feature extraction techniques using wavelet based transforms are too much complex and involves too many recursive steps. This reduces computational performance although they can offer better accuracy performance, iv) usage of supervised learning algorithms e.g. neural network, support vector machine, etc have been used to ensure better accuracy, but usage of such techniques doesn't emphasize on convergence performance as well as techniques e.g. neural network has higher dependency to perform repetitive training on trained images rather than using it from features. Such process significant cost the execution time of algorithms that has not been much discussed in existing technique. Hence, these are the open issues in existing research techniques that are yet to be solved. The next section introduced methodology to address such problems.

\section{Proposed Research Methodology}

The primary aim of the proposed system is to carry out a simple and effective classification process for assisting in understanding the state of criticality of the tumor condition for a given brain image. The proposed system considers the analytical research methodology in order to perform the implementation of the process flow as exhibited in Fig.1. The proposed system basically acts as a framework that considers the input radiological image of brain, which is further subjected to multi-stage preprocessing e.g. gamma correction, adaptive histogram equalization linear/non-linear filtering process in order to obtain superior enhancement of the input image. The significant level of features are extracted from the enhanced image in the form of binarized threshold image which is further subjected to maximum 3 level of decomposition using wavelet transforms. This process significant assists in obtaining enriched and smaller number of features in the form of decomposed coefficients. Although, the enrichment of the features are good but they are very large in number that could offer significant consumption of processing time. We address this problem by using an algorithm that performs dimension reduction. This algorithm successfully extracts the abstract of statistical data from the feature obtained in prior stage and thereby makes the data much precise with reduced size. We use Principal Component Analysis (PCA) for this purpose. The final step is to apply algorithm of classification where supervised classification technique e.g. Support Vector Machine (SVM) is utilized for categorizing the criticality state of tumor in the form of malignant or benign state of output (Fig.1).

\section{ALGORITHM DESCRIPTION}

This section discusses about the algorithms implemented in order to carry out classification of brain tumor. The proposed system initially takes the input of a brain images and subject it to a multi-level image enhancement process. This process allows performing on-demand preprocessing based on the complexity of the visualization of an image. The proposed system introduces three algorithms that perform sequential operation in order to achieve the research goal. The discussions of the algorithms are as follows: -

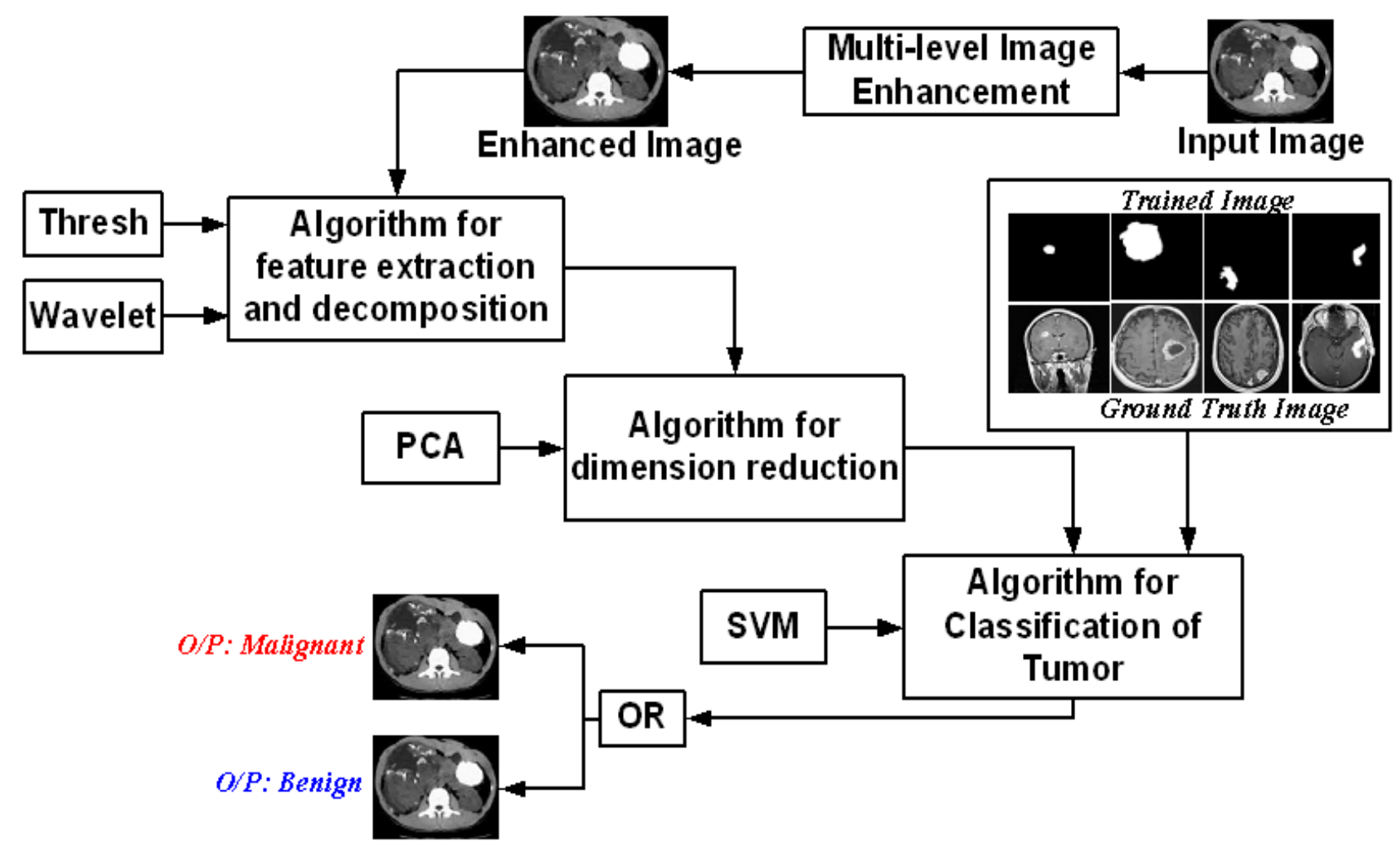

Fig. 1. Process Flow of Proposed System. 


\section{A. Algorithm for Feature Extraction and Decomposition}

This algorithm is responsible for extracting explicit set of preliminary features that will assists in classification process in later phase of implementation. Let $\mathrm{L}$ be the number of gray levels for representing a pixel in an image, the gray level ranges from $[1,2 \ldots \mathrm{L}]$. The probability distribution function for the normalized gray level histogram is given by,

$p_{i}=\frac{n_{i}}{N} \quad p_{i}>0, \sum_{i=1}^{L} p_{i}=1$

Where, $n_{i} \rightarrow$ number of pixels at the $i^{\text {th }}$ level

$\mathrm{N} \rightarrow$ Total number of pixels $\left(\mathrm{n}_{1}, \mathrm{n}_{2} \ldots \mathrm{n}_{\mathrm{L}}\right)$

The image pixel is differentiated into pixels of two classes which are identified as foreground and background objects, this is obtained by setting a threshold at level $\mathrm{k}$. The upper bound and the lower bound of the threshold is represented by $\mathrm{C}_{0}$ and $\mathrm{C}_{1}$, where, $\mathrm{C}_{0}$ ranges from 1 to $\mathrm{k}$ and $\mathrm{C}_{1}$ ranges from $\mathrm{k}+1$ to $\mathrm{L}$. The probability of occurrence is given as follows,

$w_{0}=P_{r}\left(C_{0}\right)=\sum_{i=1}^{k} p_{i}=w(k), w_{1}=P_{r}\left(C_{1}\right)=\sum_{i=1 p_{i}}^{L}=1-w(k)$

The respective mean is computed as follows,

$\mu_{0}=\sum_{i=1}^{k} i P_{r}\left(i \mid c_{0}\right)=\sum_{i=1}^{k} i p_{i} / w_{0}=\mu(k) / w(k)$

$\mu_{1}=\sum_{k+1=1}^{L} i P_{r}\left(i \mid c_{1}\right)=\sum_{i=k+1}^{L} i p_{i} / w_{1}=\mu_{\tau}-\mu(k) / 1-w(k)$

Where,

$w(k)=\sum_{i=1}^{k} p_{i}$

and

$\mu(k)=\sum_{i=1}^{k}{ }^{i} p_{i}, \quad \mu_{v}=\mu(L)=\sum_{i=1}^{L} i_{p_{i}}$

Where, $\mu_{\mathrm{v}} \rightarrow$ total mean level of the original picture. The variances are given by,

$\sigma_{0}^{2}=\sum_{i=1}^{k}\left(i-\mu_{0}\right)^{2} \operatorname{Pr}\left(i \mid C_{0}\right)=\sum_{i=1}^{k} \frac{\left(i-\mu_{0}\right)^{2} p_{i}}{\omega_{0}}$

$\sigma_{1}^{2}=\sum_{i=k+1}^{L}\left(i-\mu_{1}\right)^{2} \operatorname{Pr}\left(i \mid C_{1}\right)=\sum_{i=k+1}^{L} \frac{\left(i-\mu_{1}\right)^{2} p_{i}}{\omega_{1}}$

The following discriminant criterion measure is applied to evaluate the goodness of the threshold value,

$\lambda=\sigma_{B}^{2} / \sigma_{w}^{2}, k=\sigma^{2}{ }_{T} / \sigma_{w}^{2}, \eta=\sigma^{2}{ }_{B} / \sigma_{T}^{2}$

Where,

$\sigma^{2}{ }_{w}=\omega_{0} \sigma_{0}^{2}+\omega_{1} \sigma_{1} 2$

$\sigma_{w}^{2}=\omega_{0}\left(\mu_{0}-\mu_{\gamma}\right) 2+\omega_{1}\left(\mu_{1}-\mu_{\gamma}\right)^{2}$

$=w_{0} w_{1}\left(\mu_{1}-\mu_{0}\right)^{2}$
The discriminant criteria which maximizes $\lambda, \mathrm{k}$ and $\eta$ for $\mathrm{k}$ are however equivalent to one another, due to the following relation

$\sigma_{w}^{2}+\sigma_{B}^{2}=\sigma_{T}^{2}$

The optimal threshold $k^{*}$ which maximizes $\eta$ or $\sigma_{B}^{2}$ is selected for sequential search by using a simple cumulative search,

$\eta(k)=\sigma_{B}^{2}(k) / \sigma_{T}^{2}$

where,

$\sigma_{B}^{2}=\frac{\left[\mu_{T w}(k)-\mu(k)\right]^{2}}{w(k)[1-w(k)]}$

Optimal threshold for $\mathrm{k}^{*}$ is given by,

$\sigma_{B}^{2}(k *)=\max _{1 \leq k \leq L} \sigma_{B}^{2}(k)$

From the above optimization problem the range of $\mathrm{K}$ over which the maximum is sought can be restricted to

$S^{*}=\left\{k ; \omega_{0} \omega_{1}=\omega(k)[1-\omega(k)]>0\right.$, or $\left.0<\omega(k)<1\right\}$

The above equation is known as the effective range of gray level histogram. Converting the image from rgb to binary format using the above threshold value.The image of RGB format is transformed to binary image by considering the threshold value $\mathrm{k}$ defined above, the value at each pixel position is compared with the threshold value. The following condition is applied for the RGB to binary image transformation,

$$
I_{1}(i, j)=\left\{\begin{array}{l}
I\left(i_{x}, j_{y}\right) \leq k, \text { then } I\left(i_{x}, j_{y}\right)=0 \\
I\left(i_{x}, j_{y}\right)>k \text {, then }\left(i_{x}, j_{y}\right)=1
\end{array}\right.
$$

Where, $\mathrm{i}_{\mathrm{x}}, \mathrm{i}_{\mathrm{v}}$ is current pixel position of the image, $\mathrm{k}$ is threshold value, $l_{1}(i, j)$ is resulting Binary image, and $1(i, j)$ is Input RGB image. The next part of the study considers implementing discrete wavelet transformation as decomposition is required Consider an image $f(x, y)$ of size $\mathrm{MxN}$ whose forward discrete transform is $\mathrm{T}(\mathrm{u}, \mathrm{v}, \ldots)$ is expressed in terms of general relation as mentioned below.

$T(u, v)=\sum_{x, y} f(x, y) g_{u, v,} \ldots(x, y)$

Where, $\mathrm{x}$ and $\mathrm{y}$ are spatial variables and $\mathrm{u}, \mathrm{v}$ are transform domain variables. Given $\mathrm{T}(\mathrm{u}, \mathrm{v} \ldots), \mathrm{f}(\mathrm{x}, \mathrm{y})$ is obtained using generalized inverse discrete transform which is given below,

$f(x, y)=\sum_{u, v} T(u, v, \ldots) h_{u, v,} \ldots(x, y)$

Where, $g_{u, v}$ and $h_{u, v}$ in these equations are called forward and inverse transformation kernels respectively. By applying the Discrete Fourier Transform (DFT), in this case

$h_{u, v}(x, y)=g_{u, v}^{*}(x, y)=\frac{1}{\sqrt{M N}} e^{j 2 \Pi(u x / M+v y / N)}$ 
Where, $\mathrm{u} \rightarrow$ horizontal frequency and $\mathrm{v} \rightarrow$ vertical frequency.

The kernels are separable since

$h_{u, v}(x, y)=h_{u}(x) h_{v}(y)$

for

$h_{u}(x)=\frac{1}{\sqrt{M}} e^{j 2 \Pi u x / M}$ and $h_{v}(y)=\frac{1}{\sqrt{N}} e^{j 2 \Pi v y / N}$

and orthonormal because,

$\left\langle h_{r}, h_{s}\right\rangle=\delta_{r s}= \begin{cases}1 & r=s \\ 0 & \text { otherwise }\end{cases}$

The Daubechies wavelet is based on the work proposed by Ingrid Daubechies which belong to a family of orthogonal wavelets. It defines the discrete wavelet transform and is characterized by a maximal numbers of vanishing moments for some given support. The scaling sequence is generally represented by an orthogonal discrete wavelet transform with approximation order A

$$
a(z)=2^{1-A}(1+z)^{A} p(z)
$$

with $\mathrm{N}=2 \mathrm{~A}$ having real coefficients, $\mathrm{p}(1)=1$ and degree (p) = A-1, the orthogonality condition can be given as

$$
a(z) a\left(z^{-1}\right)+a(-z) a\left(-z^{-1}\right)=4
$$

The above equation can also be represented as,

$$
(2-x)^{A} P(X)+X^{A} P(2-X)=2^{A}
$$

The algorithm takes the input of $I$ (preprocessed image), $l$ (level of decomposition) that after processing leads to generation of feat (feature) as an output. The steps of algorithm are as follows:

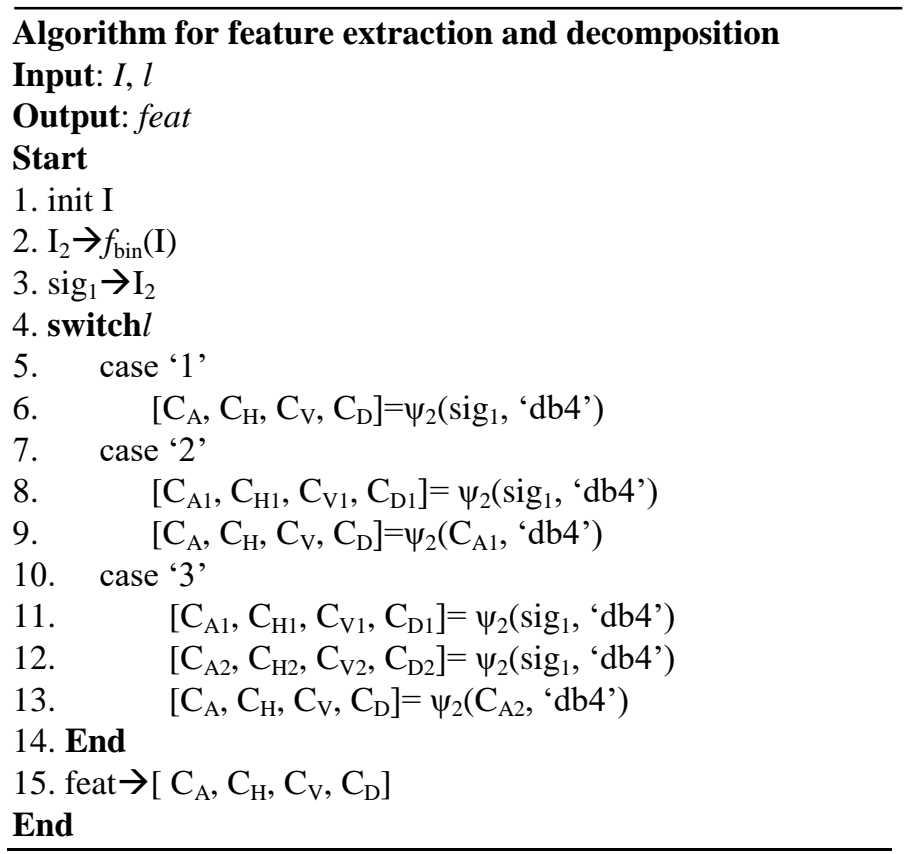

The algorithm takes the input image and digitizes it (Line1) followed by applying threshold-based binarization function $\mathrm{f}_{\text {bin }}$ (Line-2). This mechanism leads to a binarized image $\mathrm{I}_{2}$ that is further subjected to wavelet-based decomposition of 3 level $l$ (Line-4). Depending on the selected case of level of decomposition (Line-5, Line-7, and Line-10), the proposed system applying a two-dimensional wavelet function $\psi_{2}$ on signal $\operatorname{sig}_{1}$ (Line-3) i.e. binarized image. The algorithm implements Daubechies 4 (Line-4 to Line-14) for performing decomposition in order to obtain 4 different coefficients i.e. approximation coefficient $\left(\mathrm{C}_{\mathrm{A}}\right)$, horizontal coefficient $\left(\mathrm{C}_{\mathrm{H}}\right)$, vertical coefficient $\left(\mathrm{C}_{\mathrm{V}}\right)$, and diagonal coefficient $\left(\mathrm{C}_{\mathrm{D}}\right)$. Finally, a matrix is constructed feat that stores all the final features upon selection of any specific level of decomposition (Line15). The outcome is further subjected to algorithm of dimension reduction.

\section{B. Algorithm for Dimension Reduction}

This algorithm is responsible for further improving the search towards better and more accurate features. It performs minimization of dimensions of the features obtained. The purpose of implementing the principal component analysis is primarily reducing the dimensionality of the data which consists of large number of inter-correlated variables, while still maintaining the variation present in the dataset. The principal component which are uncorrelated and retains the first few variations present in all of the original variables.To derive the form of the PCs, consider, $\alpha_{1}$ xwhere, $\alpha_{1}$ maximizes $\operatorname{var}\left[\alpha_{1} \mathrm{x}\right]=\alpha_{1} \sum \mathrm{x}$. To maximize $\alpha_{1} \sum \mathrm{x}$ subject to $\alpha_{1} \alpha_{1}=1$, the standard approach is to use the technique of Lagrange multipliers. Maximize

$\alpha_{1} \sum \alpha_{1}-\lambda\left(a_{1}^{\prime} \alpha_{1}-1\right)$

Where $\lambda$ is a Lagrange multiplier. Differentiation with respect to $\alpha_{1}$ gives,

$\sum \alpha 1-\lambda \alpha_{1}=0$ or $\left(\sum-\lambda I_{p}\right) \alpha_{1}=0$

Where $I_{p}$ is the $(p \times p)$ identity matrix. Thus, $\lambda$ is an eigenvalue of $\sum$ and $\alpha_{1}$ is the corresponding eigenvector. To decide which of the $\mathrm{p}$ eigenvectors gives $\alpha_{1} \mathrm{x}$ with maximum variance, note that the quantity to be maximized is

$\alpha_{1}^{\prime} \sum a_{1}=\alpha^{\prime}{ }_{1} \lambda \alpha_{1}=\lambda \alpha_{1}^{\prime} \alpha_{1}=\lambda$

Where, $\lambda$ is very large.

In general, the $\mathrm{k}^{\text {th }} \mathrm{PC}$ of $\mathrm{x}$ is $\alpha_{\mathrm{k}}^{\prime} \mathrm{x}$ and $\operatorname{var}\left(\alpha_{\mathrm{k}}^{\prime} \mathrm{x}\right)=\lambda_{\mathrm{k}}$, where, $\lambda_{\mathrm{k}} \rightarrow \mathrm{k}^{\mathrm{th}}$ largest eigenvalue of $\sum$ and $\alpha_{\mathrm{k}}$ is the corresponding Eigen vector. The second Principal Component, $\alpha_{2} \mathrm{X}$, maximizes $\alpha_{2} \sum \alpha_{2}$ subject to being uncorrelated with $\alpha_{1} \mathrm{x}$ or equivalently subject to $\operatorname{cov}\left[\alpha_{1} \mathrm{x}, \alpha_{2} \mathrm{x}\right]=0$, where $\operatorname{cov}(\mathrm{x}, \mathrm{y})$ denotes the covariance between the random variables $\mathrm{x}$ and $\mathrm{y}$. But

$$
\begin{aligned}
& \operatorname{cov}\left[\alpha_{1}^{\prime} x, \alpha_{2}^{\prime} x\right]=\alpha_{1}^{\prime} \sum \alpha_{2}=\alpha_{2}^{\prime} \sum \alpha_{1}=\alpha_{2}^{\prime} \lambda_{1} \alpha_{1}^{\prime}= \\
& \lambda_{1} \alpha^{\prime} 2 \alpha_{1}^{\prime}=\lambda 1 \alpha_{1}^{\prime} \alpha_{2}
\end{aligned}
$$

Thus, any of the equations 
$\alpha_{1}^{\prime} \sum \alpha_{2}=0, \alpha_{2}^{\prime} \sum \alpha_{1}=0$

$\alpha_{2}^{\prime} \alpha_{2}=0, \alpha_{2}^{\prime} \alpha_{1}=0$

could be used to specify zero correlation between $\alpha_{1} \mathrm{x}$ and $\alpha_{2} \mathrm{x}$. Choosing the last of these (an arbitrary choice), and noting that a normalization constraint is again necessary, the quantity to be maximized is

$\alpha_{2}^{\prime} \sum \alpha_{1}-\lambda\left(\alpha_{2}^{\prime} \alpha_{2}-1\right)-\phi \alpha_{2}^{\prime} \alpha_{1}$

where $\lambda, \varphi$ are Lagrange multipliers. Differentiation with respect to $\alpha_{2}$ gives

$\alpha_{1}^{\prime} \sum \alpha_{2}-\lambda_{\alpha 2}-\phi \alpha_{1}=0$

and multiplication of the above equation on the left by $\alpha_{1}$ gives,

$\alpha_{1}^{\prime} \sum \alpha_{2}-\lambda \alpha_{1} \alpha_{2}-\phi \alpha_{1}^{\prime} \alpha_{1}=0$

which, since the first two terms are zero and $\alpha_{1} \alpha_{1}=1$, reduces to $\phi=0$. Therefore, $\sum \alpha_{2}-\lambda \alpha_{2}=0$, or equivalently $\left(\sum-\lambda I_{p}\right)$ $\alpha_{2}=0$, so $\lambda$ is once more an eigenvalue of $\sum$, and $\alpha_{2}$ the corresponding eigenvector. Again, $\lambda=\alpha_{2}^{1} \sum \alpha_{2}$, so $\lambda$ is to be as large as possible. Assuming that $\sum$ does not have repeated eigenvalues. If it did, it follows that $\alpha_{2}=\alpha_{1}$, violating the constraint $\alpha_{1}{ }_{1} \alpha_{2}=0$. Hence $\lambda$ is the second largest eigenvalue of $\sum$, and $\alpha_{2}$ is the corresponding eigenvector. As stated above, it can be shown that for the third, fourth, ..., $\mathrm{p}^{\text {th }}$ PCs, the vectors of coefficients $\alpha_{3}, \alpha_{4}, \ldots, \alpha_{\mathrm{p}}$ are the eigenvectors of $\sum$ corresponding to $\lambda_{3}, \lambda_{4}, \ldots . \lambda_{\mathrm{p}}$, the third and fourth largest and the smallest eigenvalue, respectively. Furthermore, $\operatorname{var}\left[\alpha_{\mathrm{kx}}{ }^{\prime}\right]=\lambda_{\mathrm{k}}$ for $\mathrm{k}=1,2, \ldots \mathrm{p}$. The vectors $\alpha_{\mathrm{k}}$ are referred to as principal components. The algorithm takes the input of obtained feat (decomposed feature) from first algorithm and gives the output of feat $t_{\text {red }}$ (features with reduced dimension). The significant steps of the algorithm are as follows:

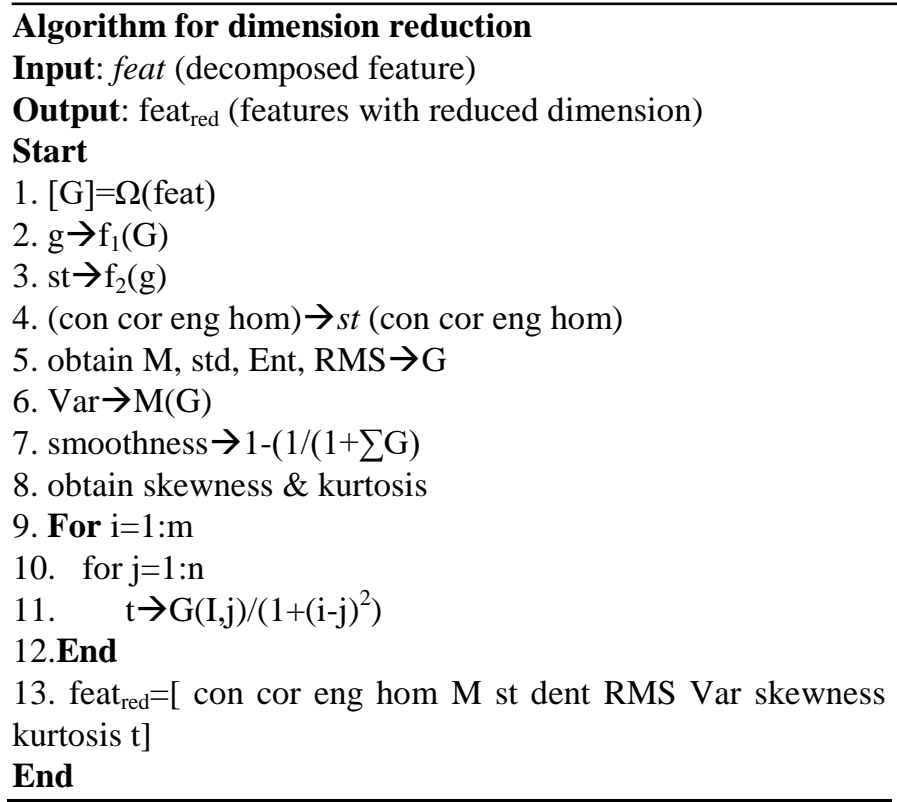

The algorithm implements an orthogonal transformation function of principal component analysis $\Omega$ on the obtained feature feat from first algorithm (Line-1). Applying the function $\Omega$ results in a coefficient G (Line-1) that is further used for constructing a new occurances matrix of gray level i.e. by using $f_{1}$ function (Line-2). This process results in a new variable $g$ (Line-2). A consecutive function $\mathrm{f}_{2}$ is applied on obtained variable $\mathrm{g}$ for normalizing the obtained matrix (Line3 ). This process results in generation of matrix with summation 1 and thereby dimensionality is reduced to greater extent. The obtained statistical information st (Line-3) can now be said to represent the properties of the matrix that can further extract contrast con, correlation cor, energy eng, and homogeneity hom (Line-4). The next step of the algorithm is to obtain mean $M$, standard deviation $s t d$, entropy Ent, and root mean square RMS (Line-5). More statistical information is obtained e.g. variance Var, smoothness, kurtosis, and skewness (Line-8) from the matrix G (Line-6 to Line-8). Considering all the rows and columns of matrix $\mathrm{G}$, a temporary feature $t$ is obtained (Line-11). Finally, the outcome of feature with reduced dimensionality is computed from all the above obtained metrics (Line-13). This process reduces the redundant information and retains enriched statistical pixel data that significant enrich the feature obtained from first algorithm.

\section{Algorithm for Classification of Tumor}

The proposed system uses supervised learning algorithm in order to perform classification. We use Support Vector Machine for this purpose. The primary purpose of SVM method is to identify and classify complex patterns present in the data. There are two approaches to this problem, the first approach is a computational approach and the second is the statistical approach. The basic principle of classification is that the class of kernel methods implicitly defines class of possible patterns by introducing a notion of similarity between the data. The choice of similarity is considered by the choice of relevant features. General consideration is that kernel methods use information about the inner products between the data items. Hence the kernel functions could be defined as inner product of some feature space which is potentially very complex. Therefore, the necessity of specifying the features becomes lesser when the kernel is given. The first feature of the support vector machines corresponds to that of the duality, i.e. SVM's are known to be linear learning machines which are represented in a dual fashion

$$
f(x)=\langle w, x\rangle+b=\sum \alpha_{i} y_{i}\left\langle x_{i} x\right\rangle+b
$$

Where, $\mathrm{w}=\sum \alpha_{\mathrm{i}} \mathrm{y}_{\mathrm{i}} \mathrm{x}_{\mathrm{i}}$, if $\alpha_{\mathrm{i}}>0 \rightarrow$ linear combination of training points. The data appears only within the dot products (in decision function and training algorithm). The update rule in dual representation is given as follows i.e.

$$
y i\left(\sum \alpha_{j} y_{j}\left\langle x_{j}, x_{i}\right\rangle+b\right) \leq 0 \text { Then, } \alpha_{i} \leftarrow \alpha_{i}+\eta
$$

It is to be noted that the data appears only inside the dot products in dual representations. Hence, a closer look into the technique adopted here shows that it has an inclusion of regularization attribute that significantly assist in over-fitting problems in the classification of brain tumor. From the computational complexity viewpoint, it was already seen that 
existing machine learning approaches suffers from it. However, proposed system addresses no local minima by solving the convex optimization problem. This offers the algorithm not only to obtain the convergence performance in lowest rounds but also in each rounds the algorithm offers significant performance improvement in terms of accurate classification process. This is the final algorithm that perform classification of the given brain tumor image. The algorithm takes the input

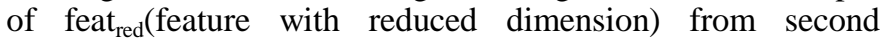
algorithm and gives the output of classification of tumor as malignant/benign case. The steps involved in the proposed algorithm are as follows:

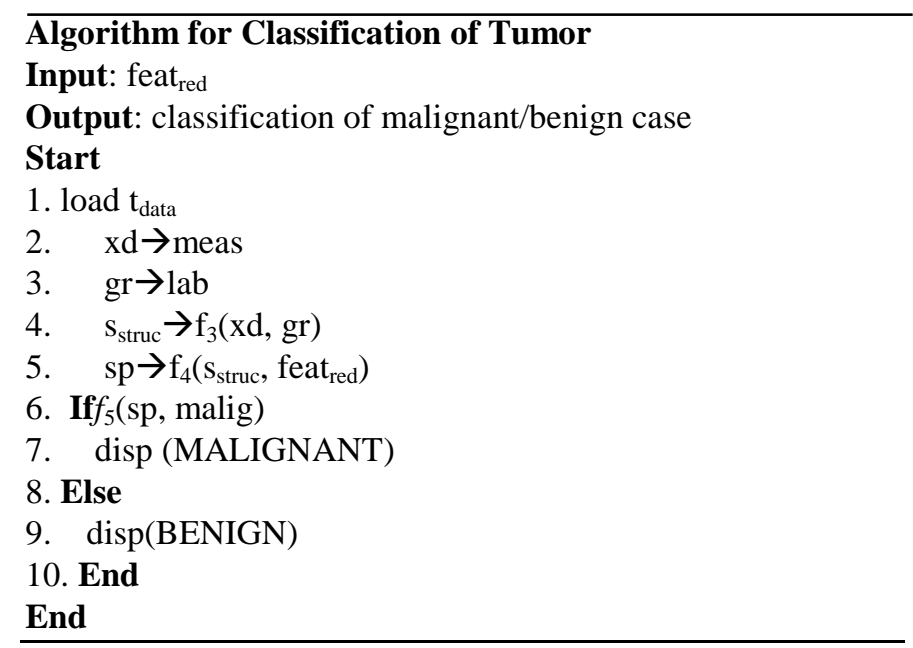

The preliminary step of this algorithm is to load the trained data $t_{\text {data }}$ (Line-1). The algorithm access the elements of the table using the variable meas assigned to matrix $x d$ (Line-2) as well as it also assign a label lab to the group matrix $g r$ (Line3 ). The algorithm implements a support vector machine in the form of function $\mathrm{f}_{3}$ on $x d$ and $g r$ that gives the output in the form of structure $s_{\text {truck }}$ (Line-4). This new structure $s_{\text {truck }}$ consists of all the relevant data associated with supervised trained classifier. The system also uses dot product as the linear kernel function. The next part of the algorithm is to perform classification using function $\mathrm{f}_{4}$ (Line5). This function assists in categorizing the data existing in the row of matrix feature of reduced dimension feat ${ }_{\text {red }}$ using the data of trained classifier structure. We use a comparative function $f_{5}$ in order to check the comparison among all the classifiers i.e. $s p$ (Line-6). A true comparison will result in finding the malignant case of tumor (Line-7) or else the benign case is confirmed (Line-9).

It should be known that all the illustrated three algorithms performs sequential operation where the algorithm performs the run-time execution and doesn't store anything on its memory. This process is carried out by constructing a temporary memory of restricted minimal size that stores the run-time process and eliminates them once the results are displaced. This properties offers significant freedom from any form of storage complexity as well as lower dependencies. Hence, the proposed algorithm has better assurance of computational performance apart from accuracy in the classification performance. The next section discusses about the results obtained by implementing algorithm.

\section{RESUlt OBTAINED}

The algorithms discussed in prior section have been assessed using dataset of brain tumor images. The scripting of the proposed logic was designed using numerical computing MATLAB on normal 32 bit windows machine. The dimensions of the images are approximately in the range of $225 \times 225$. Upon taking the input image of brain tumor (Fig.2), the proposed system can undertake multiple forms of preprocessing algorithm in order to perform image enhancement. Fig.3 highlights the enhanced version of the input image.

The proposed algorithm also implements a threshold based scheme for the purpose of performing clustering of image pixels on the gray scale processed image. It significantly minimizes the extent of gray scale that further assist in segmentation process of binary image as shown in Fig.4

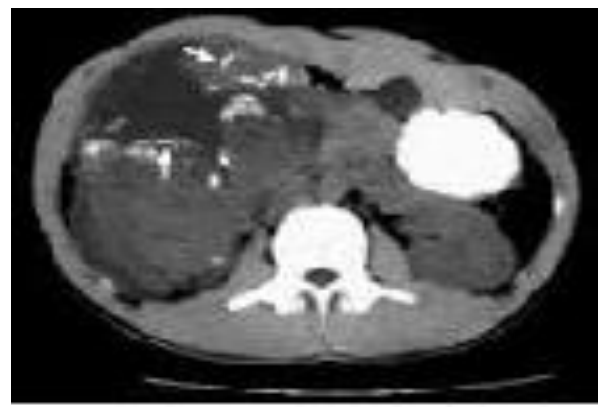

Fig. 2. Input Image of Brain Tumor.

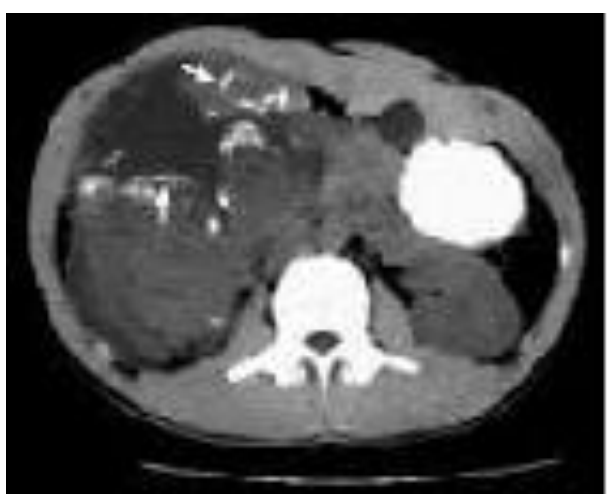

Fig. 3. Preprocessed Image.

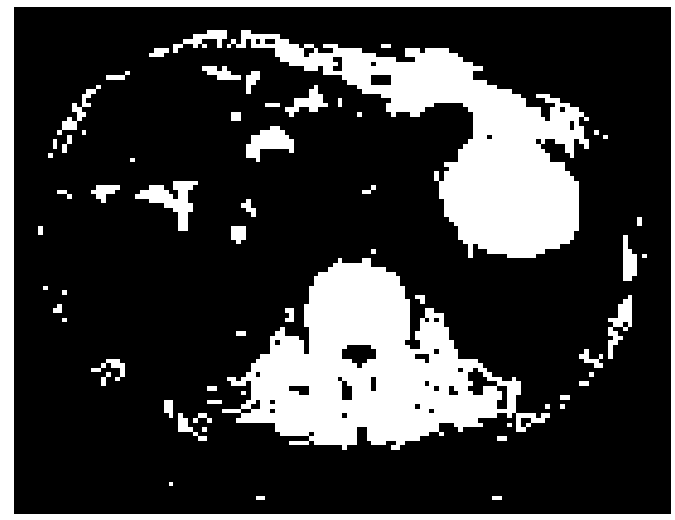

Fig. 4. Threshold Image. 
The proposed system uses threshold image information as the preliminary feature for further subjecting to be decomposed. After the thresholding of the image is carried out, the next step was to obtain the decomposition of the image using discrete wavelet transform. The proposed system uses Daubechies approach in order to perform decomposition supportable up to 3 levels. Fig.5 highlights the visuals of all the three levels of decomposition to be considered for further dimensional reduction.
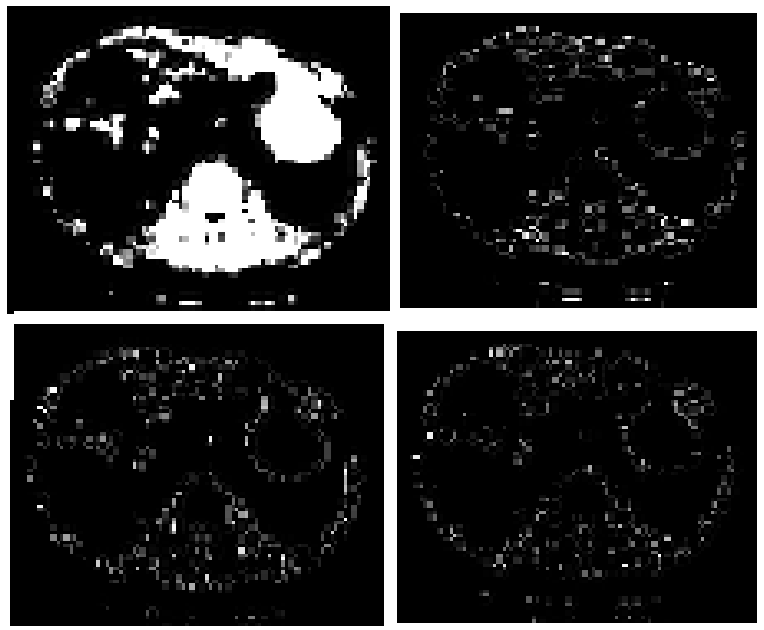

(a) Level-1 Decomposition
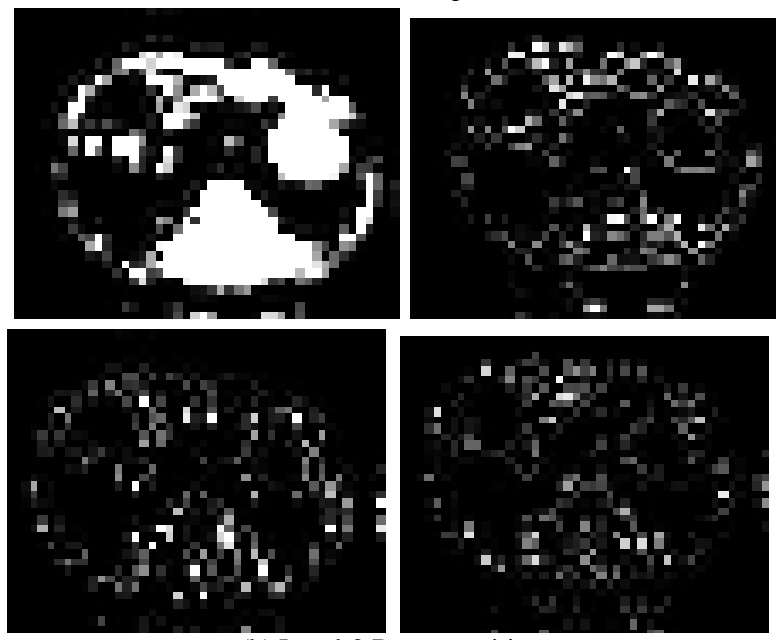

(b) Level-2 Decomposition
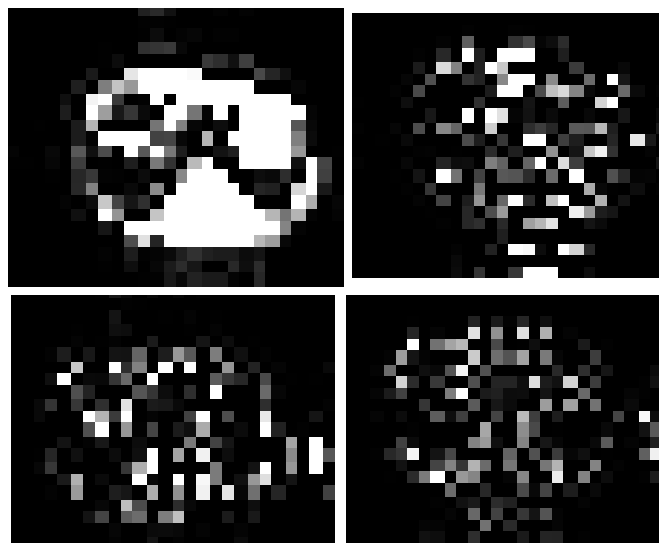

(c) Level-3 Decomposition

Fig. 5. Decomposed Image.

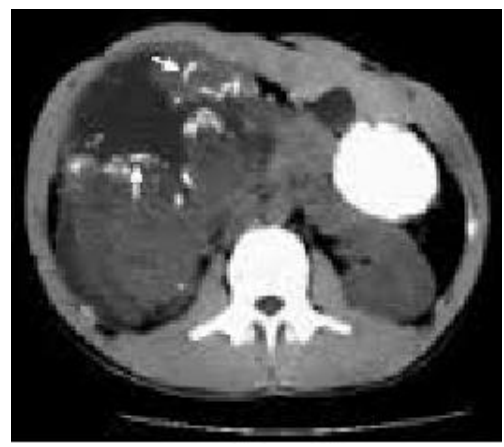

Fig. 6. Final Classified Image.

The decomposed image is further subjected to principal component analysis using algorithm-2, where the prominent intention is to retrieve certain statistical information e.g. mean, standard deviation, entropy, root mean square value, variance, smoothness, kurtosis, skewness, contrast, correlation, energy, and homogeneity. All these statistical data are computed and easy to assess owing to its numerical significance with the probability theory. Finally supervised algorithm-3 is implemented in order to obtain the classified image to be malignant (or else benign otherwise) (Fig.6). The average time taken in order to perform classification is approximately 0.36551 seconds on core $\mathrm{i} 7$ processor.

The study outcome of the proposed system is also benchmarked considering various forms of the machine learning techniques that assists in performing classification with respect to kernel e.g. quadrature, neural network, polynomial, linear, and radial basis function. The numerical assessment of the study was carried out with respect to sensitivity, specificity, precision, and accuracy.

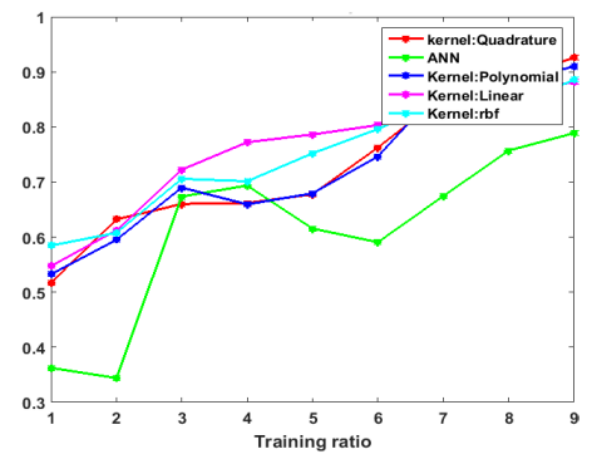

Fig. 7. Analysis of Sensitivity.

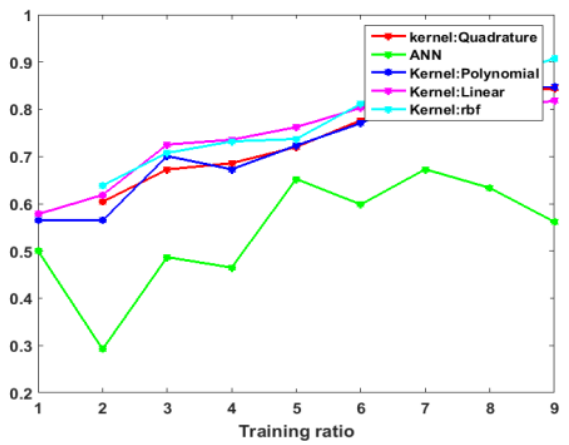

Fig. 8. Analysis of Specificity. 


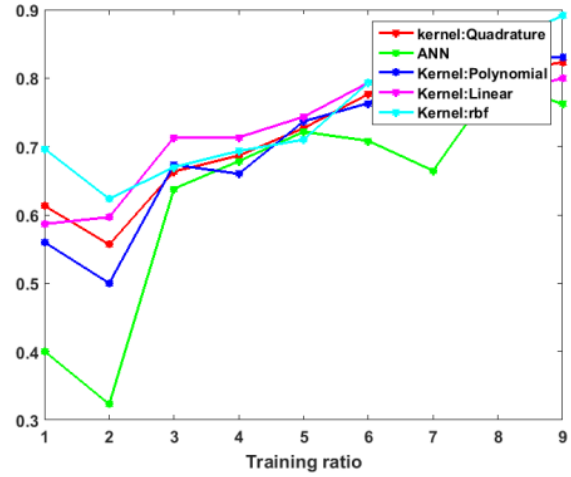

Fig. 9. Analysis of Precision.

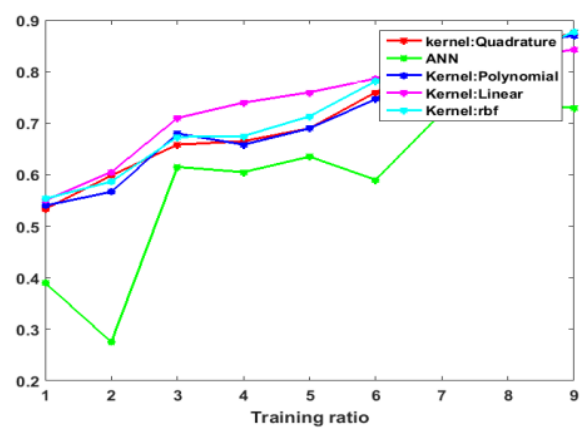

Fig. 10. Analysis of Accuracy.

Fig.7 highlights the sensitivity analysis or the true positive factor for the image queried and trained image within the database. It shows that sensitivity for linear-based kernel shows better performance altogether. This trend is followed by radialbasis function. However, there is no significant difference is found between the quadrature-based and polynomial based machine learning mechanism. The outcome directly infers that better identification as well as classification can be carried out using linear-based kernel system for the machine learning approach. The overall performance of neural network is not optimal and there are various fluctuating points owing to failure in exploring better match of elite result with activation function. Moreover achieving convergence is too slow in neural network for the given scenario and hence we recommend insufficiency of neural network in order to perform classification. Nearly similar performance can be seen in the specificity trends in Fig.8 i.e. true negative factor. Although, the specificity trend of neural network is low, but is not much recommended owing to time consumption in the classification process which is definitely not scalable. Although, the trend of linear-based kernel is found little increasing in beginning, but it is found to lower down the true negatives at the end to a large extent. The performance of polynomial and quadrature is nearly similar just like that of sensitivity. The precision and accuracy curve is shown in Fig.9 and Fig.10 which is also in the agreement with sensitivity and specificity curve. The overall performance of linear-based kernel system is found to offer higher precision and accuracy, while the trend for quadrature and polynomial remains the same. Therefore, the inference of the entire numerical benchmarked outcome is thatSupport Vector Machine definitely proves as a best classifier in comparison to the frequently used neural network.Compared to existing ANN approaches, the proposed Kernel approaches have better sensitivity (ranging between $87-93 \%$ ), specificity (ranges $82-92 \%$ ), precision (of 80\%-85) and accuracy (of 87\%88\%) approx. Thus the Kernel approaches are significant than ANN approaches (sensitivity of $79 \%$, specificity of $57 \%$, precision of $75 \%$ and accuracy of $72 \%$ ).It is also now proven that there is significant difference in adoption of 4 different kernel system of support vector machine, where linear-based kernel has proven to offer better classification performance for complex forms of brain tumor images. Also, recommend for usage of polynomial and radial basis function that also offers better significance towards accuracy performance when it comes to classification problems. Therefore, the proposed system offers a simple but yet novel classification techniques using supervised learning approach for efficient binary categorization of malignancy and benign state of brain tumor.

\section{VII.CONCLUSION}

There are various schemes implemented for enhancing the classification performance towards identifying the malignancy or benign state of brain tumor. However, existing systems are also associated with research problems that lead to start of proposed investigation. We find that it is essential to emphasize on the preprocessing that can potentially lead to minimization of the many effort of accurate classification in the latter stage. We also believe that different preprocessing has different effect on classification performance. However, at present, we generalize this preprocessing and then emphasize on the latter stage of it. The preprocessed image is subjected to binarized thresholding scheme followed by applying of wavelet transform in order to accomplish more number of enriched features. We further apply simple process of dimensional reduction followed by supervised learning. The study outcome shows different forms of performance on different form of kernel system and is found to be much better in comparison to existing technique of neural network.

The future researches can consider this work as base for classifying the malignancy and benign state of brain tumor by using supervised learning approach which may bring higher accuracy in classification of tumor. Also, this work can be considered with diagnosis time, and sensitivity for brain MRI classification.

\section{ACKNOWLEDGEMENT}

I thank Dr. Rajshekar M, radiologist who helped me in carrying out research work by giving real time images.

\section{REFERENCES}

[1] E. Hattingen, U. Pilatus, Brain Tumor Imaging, Springer, 2015

[2] R. R. Colen, Imaging of Brain Tumors, An Issue of Magnetic Resonance Imaging Clinics of North America, E-Book, Elsevier Health Sciences, 2016

[3] M. M Kaila, R. Kaila, Quantum Magnetic Resonance Imaging Diagnostics of Human Brain Disorders, Elsevier, 2010

[4] J. Hodler, G. K. von Schulthess, C. L. Zollikofer, Diseases of the Brain, Head \& Neck, Spine, Springer Science \& Business Media, 2012

[5] H. Huang, J. P. Owen, P. Mukherjee, Neuroanatomy of Human Brain Development, Frontiers Media, 2017

[6] J. Sachdeva, V. Kumar, I. Gupta, N. Khandelwal and C. K. Ahuja, "Multiclass Brain Tumor Classification Using GA-SVM," 2011 Developments in E-systems Engineering, Dubai, 2011, pp. 182-187. 
[7] R. Sonavane and P. Sonar, "Classification and segmentation of brain tumor using Adaboost classifier," 2016 International Conference on Global Trends in Signal Processing, Information Computing and Communication (ICGTSPICC), Jalgaon, 2016, pp. 396-403

[8] L. Farhi and A. Yusuf, "Comparison of brain tumor MRI classification methods using probabilistic features," 2017 13th IASTED International Conference on Biomedical Engineering (BioMed), Innsbruck, Austria, 2017, pp. 55-62

[9] H. B. Nandpuru, S. S. Salankar and V. R. Bora, "MRI brain cancer classification using Support Vector Machine," Electrical, Electronics and Computer Science (SCEECS), 2014 IEEE Students' Conference on, Bhopal, 2014, pp. 1-6

[10] M. Sornam, M. S. Kavitha and R. Shalini, "Segmentation and classification of brain tumor using wavelet and Zernike based features on MRI," 2016 IEEE International Conference on Advances in Computer Applications (ICACA), Coimbatore, 2016, pp. 166-169.

[11] N. B. Bahadure, A.K. Ray and H. P. Thethi, "Research Article Image Analysis for MRI Based Brain Tumor Detection and Feature Extraction Using Biologically Inspired BWT and SVM", Hindawi International Journal of Biomedical Imaging, pp. 12, 2017

[12] J. Cong, B. Wei, Y. He, Y. Yin and Y. Zheng, "A Selective Ensemble Classification Method Combining Mammography Images with Ultrasound Images for Breast Cancer Diagnosis", Computational and Mathematical Methods in Medicine, pp. 7, 2017

[13] I. I. Esener, S. Ergin, and T. Yuksel, "Research Article A New Feature Ensemble with a Multistage Classification Scheme for Breast Cancer Diagnosis", Journal of Healthcare Engineering, pp. 15, 2017

[14] M. M. Jadoon, Q. Zhang, I. U1 Haq, S. Butt, and A. Jadoon, "Research Article Three-Class Mammogram Classification Based on Descriptive CNN Features", BioMed Research International, pp. 11, 2017

[15] D. Ravì, H. Fabelo, G. M. Callic and G. Z. Yang, "Manifold Embedding and Semantic Segmentation for Intraoperative Guidance With Hyperspectral Brain Imaging," in IEEE Transactions on Medical Imaging, vol. 36, no. 9, pp. 1845-1857, Sept. 2017.

[16] Q.Z. Song, L. Zhao, X.K. Luo, and X.C.Dou, "Research Article Using Deep Learning for Classification of Lung Nodules on Computed Tomography Images", Journal of Healthcare Engineering, pp. 7, 2017

[17] T. H. Vu, H. S. Mousavi, V. Monga, G. Rao and U. K. A. Rao, "Histopathological Image Classification Using Discriminative FeatureOriented Dictionary Learning," in IEEE Transactions on Medical Imaging, vol. 35, no. 3, pp. 738-751, March 2016.

[18] M. Xi, J. Sun, L. Liu, F. Fan and X. Wu, "Research Article Cancer Feature Selection and Classification Using a Binary Quantum-Behaved Particle Swarm Optimization and Support Vector Machine", Computational and Mathematical Methods in Medicine, pp. 9, 2016

[19] L. Zhao and K. Jia, "Research Article Multiscale CNNs for Brain Tumor Segmentation and Diagnosis", Hindawi Publishing Corporation Computational and Mathematical Methods in Medicine, pp. 7, 2016

[20] K. D.Marcomini, A.A. O. Carneiro and H. Schiabel1, "Research Article Application of Artificial Neural Network Models in Segmentation and Classification of Nodules in Breast Ultrasound Digital Images", Hindawi Publishing Corporation International Journal of Biomedical Imaging, pp. 13, 2016

[21] C. Barbalata and L. S. Mattos, "Laryngeal Tumor Detection and Classification in Endoscopic Video," in IEEE Journal of Biomedical and Health Informatics, vol. 20, no. 1, pp. 322-332, Jan. 2016.

[22] V. Anitha and S. Murugavalli, "Brain tumour classification using twotier classifier with adaptive segmentation technique," in IET Computer Vision, vol. 10, no. 1, pp. 9-17, 22016.

[23] T. Kaur, B. S. Saini and S. Gupta, "Quantitative metric for MR brain tumour grade classification using sample space density measure of analytic intrinsic mode function representation," in IET Image Processing, vol. 11, no. 8, pp. 620-632, 82017.

[24] S. L. Jui et al., "Brain MRI Tumor Segmentation with 3D Intracranial Structure Deformation Features," in IEEE Intelligent Systems, vol. 31, no. 2, pp. 66-76, Mar.-Apr. 2016.

[25] S. Huda, J. Yearwood, H. F. Jelinek, M. M. Hassan, G. Fortino and M. Buckland, "A Hybrid Feature Selection With Ensemble Classification for Imbalanced Healthcare Data: A Case Study for Brain Tumor Diagnosis," in IEEE Access, vol. 4, no. , pp. 9145-9154, 2016.

[26] C-M. Svensson, R. Hübler and M.T. Figge, "Research Article Automated Classification of Circulating Tumor Cells and the Impact of Interobsever Variability on Classifier Training and Performance", Hindawi Publishing Corporation Journal of Immunology Research, pp. 9, 2015

[27] R. R. Sharma and P.Marikkannu, "Research Article Hybrid RGSA and Support Vector Machine Framework for Three-Dimensional Magnetic Resonance Brain Tumor Classification", Hindawi Publishing Corporation e Scientific World Journal, pp. 14, 2015

[28] A. Chaddad, "Research Article Automated Feature Extraction in Brain Tumor by Magnetic Resonance Imaging Using Gaussian Mixture Models", Hindawi Publishing Corporation International Journal of Biomedical Imaging, pp. 11, 2015

[29] I. Bukovsky, N. Homma, K. Ichiji, M. Cejnek, M. Slama, P.M. Benes and J. Bila, "Research Article A Fast Neural Network Approach to Predict Lung Tumor Motion during Respiration for Radiation Therapy Applications", Hindawi Publishing Corporation BioMed Research International, pp. 13, 2015

[30] M. E. Acevedo, M. A. Acevedo and F. Felipe, "Research Article Classification of Cancer Recurrence with Alpha-Beta BAM", Hindawi Publishing Corporation, Mathematical Problems in Engineering, pp. 14, 2009

[31] K. Kothavari, B. Arunadevi, and S. N. Deepa, "Research Article A Hybrid DE-RGSO-ELM for Brain Tumor Tissue Categorization in 3D Magnetic Resonance Images", Hindawi Publishing Corporation Mathematical Problems in Engineering, pp. 14, 2014

[32] X. Chen and C. Jian, "A tumor classification model using least square regression," 2014 10th International Conference on Natural Computation (ICNC), Xiamen, 2014, pp. 753-758.

[33] M. Huang, W. Yang, Y. Wu, J. Jiang, W. Chen and Q. Feng, "Brain Tumor Segmentation Based on Local Independent Projection-Based Classification," in IEEE Transactions on Biomedical Engineering, vol. 61, no. 10, pp. 2633-2645, Oct. 2014

[34] M. Huml, R. Silye, G. Zauner, S. Hutterer and K. Schilcher, "Research Article Brain Tumor Classification Using AFM in Combination with Data Mining Techniques", Hindawi Publishing Corporation BioMed Research International, pp. 11, 2013

[35] J. J. Corso, E. Sharon, S. Dube, S. El-Saden, U. Sinha and A. Yuille, "Efficient Multilevel Brain Tumor Segmentation With Integrated Bayesian Model Classification," in IEEE Transactions on Medical Imaging, vol. 27, no. 5, pp. 629-640, May 2008.

[36] M. Saddique, J. H. Kazmi and K. Qureshi, "Research Article A Hybrid Approach of Using Symmetry Technique for Brain Tumor Segmentation", Hindawi Publishing Corporation Computational and Mathematical Methods in Medicine, pp. 10, 2014

[37] A. P. Nanthagopal and R. Sukanesh, "Wavelet statistical texture features-based segmentation and classification of brain computed tomography images," in IET Image Processing, vol. 7, no. 1, pp. 25-32, February 2013.

[38] Z. L. Sun, C. H. Zheng, Q. W. Gao, J. Zhang and D. X. Zhang, "Tumor Classification Using Eigengene-Based Classifier Committee Learning Algorithm," in IEEE Signal Processing Letters, vol. 19, no. 8, pp. 455458, Aug. 2012.

[39] D. F. Brougham, G. Ivanova, M. Gottschalk, D.M. Collins, A. J. Eustace, R. O'Connor and J. Havel5, "Research Article Artificial Neural Networks for Classification inMetabolomic Studies ofWhole Cells Using $1 \mathrm{H}$ NuclearMagnetic Resonance", Hindawi Publishing Corporation, Journal of Biomedicine and Biotechnology, pp. 8, 2011

[40] C. H. Zheng, T. Y. Ng, L. Zhang, C. K. Shiu and H. Q. Wang, "Tumor Classification Based on Non-Negative Matrix Factorization Using Gene Expression Data," in IEEE Transactions on NanoBioscience, vol. 10, no. 2, pp. 86-93, June 2011.

[41] P. Asthana and S. Vashisth, "Segmentation of brain tumor from MRI images," 2017 International Conference on Computing and Communication Technologies for Smart Nation (IC3TSN), Gurgaon, 2017, pp. 262-266. 